\title{
The time calibration system for the LHAASO-WCDA experiment
}

\section{Bo Gao}

Insitute of High Energy Physics, Chinese Academy of Sciences, Beijing, China

E-mail: gaobo@ihep.ac.cn

\section{Mingjun Chen}

Insitute of High Energy Physics, Chinese Academy of Sciences, Beijing, China

E-mail: mjchen@ihep.ac.cn

\section{Zhiguo Yao}

Insitute of High Energy Physics, Chinese Academy of Sciences, Beijing, China

E-mail: yaozg@ihep.ac.cn

\section{Jinyan Liu}

University of Nankai, Tianjin, China

E-mail: jyliu@ihep.ac.cn

The Water Cherenkov Detector Array (WCDA), which is one of the main components of the Large High Altitude Air Shower Observatory (LHAASO), functions as surveying the northern sky for high-energy gamma ray sources at the energy range around $100 \mathrm{GeV}-30 \mathrm{TeV}$. The precision of the time measurement for shower particles hitting every detector in the array directly associates the detection sensitivity for the sources. In order to make the pointing error of the detector to any point source less than $0.1^{\circ}$, the calibration precision of the time offsets among detector cells should be less than 0.2 ns. To reach this goal, a method called "cross calibration" is to be employed for the detector array, with 180 bundles of fiber systems guiding the lights of LEDs to each PMT. A prototype of the time calibration system is developed, which is based on a fast LED, a plastic fiber bundle and an FPGA control board. The prototype is tested in the lab and the error propagation based on the measured parameters shows that it fulfills the requirement.

35th International Cosmic Ray Conference - ICRC2017

10-20 July, 2017

Bexco, Busan, Korea 


\section{Introduction}

The Large High Altitude Air Shower Observatory (LHAASO) project is located in Daocheng, Sichuan Province, China, $4410 \mathrm{~m}$ a.s.1.. The critical physical goals of LHAASO are searching for origin of high-energy cosmic ray, the evolution of the universe, high-energy celestial evolution and dark matter [1]. To achieve these goals, the proposed project consists of combined multiple air shower detection techniques, i.e., $1 \mathrm{KM}^{2}$ extensive air shower Array (KM2A), Water Cherenkov Detector Array (WCDA) and the Wide Field-of-view Cherenkov Telescope Array (WFCTA), for joint observation and eventually to the precise measurement of the cosmic ray energy spectrum and ingredients [2].

As one of the major components in LHAASO, WCDA covers an area of 78,000 $\mathrm{m}^{2}$ and consists of three water pools separated into 3,120 detector cells, as it is shown in Fig. 1. The size of two pools is $150 \mathrm{~m} \times 150 \mathrm{~m}$ each, and the third pool is $300 \mathrm{~m} \times 110 \mathrm{~m}$. For each cell, a large photomultiplier tube (PMT) with the photocathode upward is placed on the bottom center. The PMTs are used to receive Cherenkov photons which produced by cosmic ray shower secondary particles in water [3]. The main purpose of the WCDA is to survey the northern sky for VHE gamma ray sources. The detector efficiency is a crucial factor for obtaining a high sensitivity; real time monitoring and accurate calibration of the detector, on the other hand, is of importance for achieving good spectrum measurement to the targeted gamma ray sources [4].

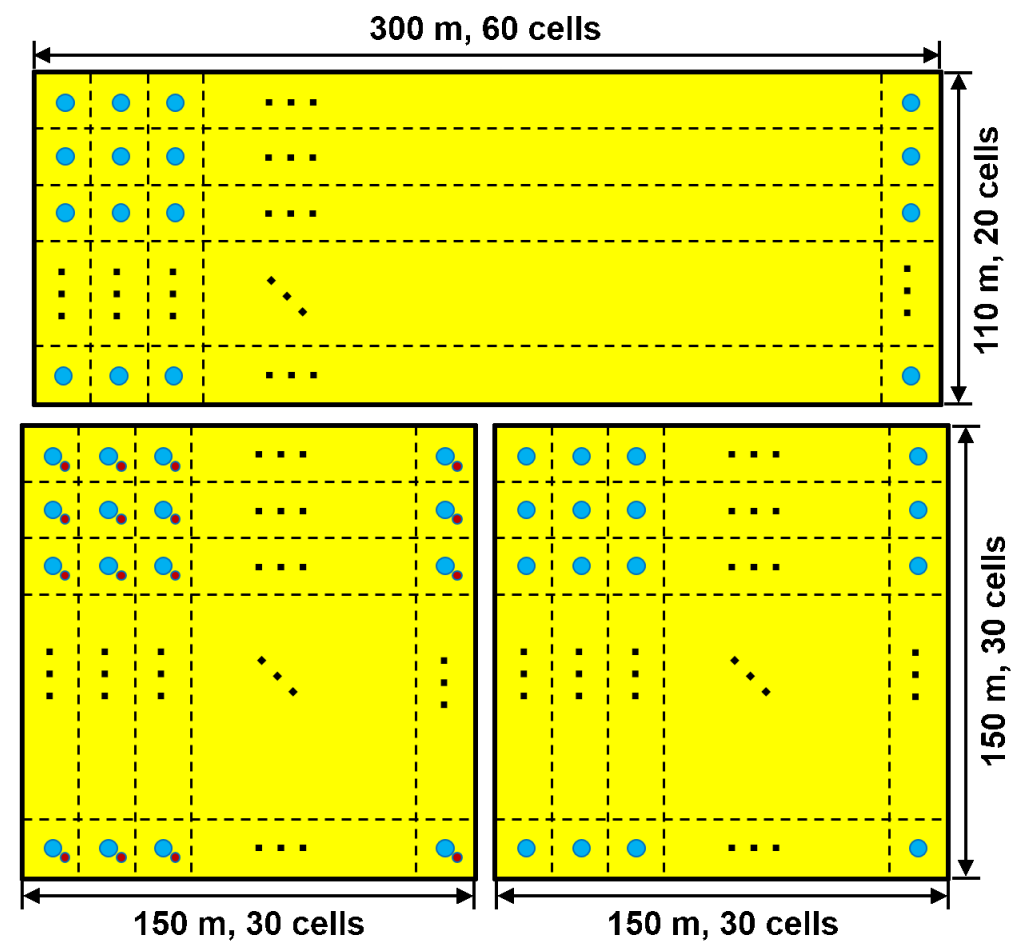

Figure 1: Schematic of WCDA layout.

For the ground based particle detector array, the shower front is reconstructed by the arrival time of Cherenkov photons produced by shower in water via triggered PMTs. The angle reconstruc- 
tion precision and error of original particle direction largely depend on the calibration precision of PMT channels, and the variation of time offsets during the operation of detector. Therefore, a precise and stable time calibration system is necessary [5].

\section{Time calibration system}

\subsection{Requirement of calibration precision}

LHAASO-WCDA requires that the radiation direction reconstruction error is better than $0.1^{\circ}$ [6]. Assuming that 1$)$ in $36(6 \times 6)$ detector cells (called a cluster), 20 detector units are triggered by a low energy shower; 2) the time offsets between the average value of the calibration unit error is $d T ; 3)$ the distance between the detector and shower axis is $L=7.5 \mathrm{~m}$ for geometric assumptions, then $d T$ must satisfy:

$$
\frac{(d T \times c)}{(L \times \sqrt{20-3})} \leqslant \frac{0.1}{57.3}
$$

Where $c$ is the speed of light, and (20-3) is the degrees of freedom of plane fitting, which needs 3 parameters to fitting: zenith angle, azimuth angle and the arrival time of first arrival particle. By calculating the $d T$ approximation of $0.18 \mathrm{~ns}$, then the time calibration accuracy is defined as half of reconstruction error, i.e., $\delta T=1 / 2 d T=0.09 \mathrm{~ns}$.

\subsection{Calibration method}

Because the neighbour detector cells are partioned by the black plastic curtains to prevent the cross-talk of Cherenkov photons, it's not appropriate to calibrate the whole array by using one single light source [7]. Combined with the layout WCDA electronics system, the design of the time calibration system is also based on the concept of "cluster", which contains 36 detector cells. Two sets of light source are used in one cluster, each light source includes one blue LED and one plastic optical fiber bundle, each fiber bundle contains 40 optical fibers and 4 of them are the backup. For one set, 36 optical fibers are used to calibrate the time offsets among internal channels. For the other set, 20 optical fibers are used to calibrate the internal part of the PMT channels, the other 16 optical fibers are used to calibrate the four neighbor clusters' PMT channels, each neighbor cluster uses 4 channels for cross calibration. Two sets of the light source are alternately powered by the remote control system. For the time calibration of 3 pools, it can be achieved by using the shower events which cover any 2 or all 3 pools. Fig. 2 is the scheme of the deployment of the calibration system. For example, one small pool need 50 sets of calibration. With this configuration, 180 sets of time calibration devices are needed in the whole WCDA. In the future calibration system work conditions, the calibration light intensity for hundreds of PE. In order not to affect the operation of the shower, the LED to take low-frequency and continuous calibration method.

\section{Fibers' test platform}

\subsection{Introduction to the setup}

\subsubsection{The light source}

As mentioned above, each set of calibration device includes one LED and 40 optical fibers. 


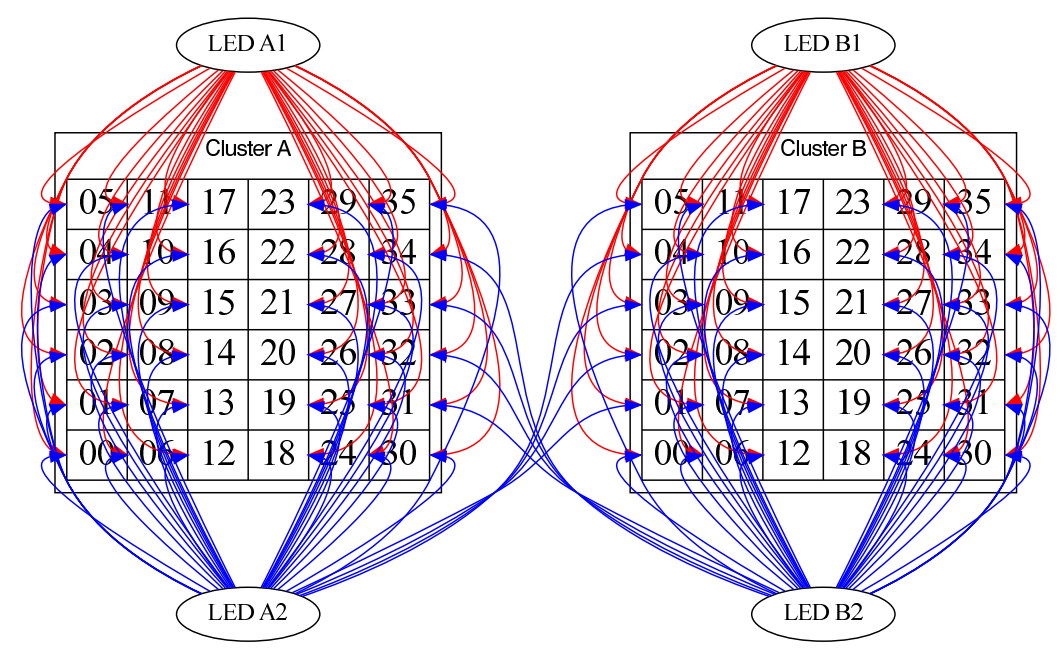

Figure 2: Schematic of time cross calibration.

The peak wavelength of LED is $468 \mathrm{~nm}$ and the viewing angle is $120^{\circ}$. Light guide tube is made of 304 stainless steel with an inner diameter of $20 \mathrm{~mm}$ and black oxide surface treatment. The LED fixed at one end of the tube with soldering one BNC connector, facing forwards to the fiber bundle end. The LED tub output uniformity is better than $\pm 2 \%$. Furthermore, the LED is stable and precision is good enough to do the time calibration. The core diameter of each plastic optical fiber is $1.0 \mathrm{~mm}$, the optical attenuation length is $26 \mathrm{~m}$ under the light wavelength $468 \mathrm{~nm}$. One end of the 40 optical fibers are glued together in a round shape and fine polished, the other end of each fiber is mounted by SMA905 stainless steel fiber connector, all the fibers are numbered for test in the lab and installation in the WCDA.

\subsubsection{Test}

platform The test platform consists of two parts: one-dimensional stepper platform and two fast PMTs[8].

The one-dimensional platform consists of one motor, screw rod, slide fiber brackets and PMT brackets. The length of screw rod is $110 \mathrm{~cm}$. The length of aluminum fiber bracket is $710 \mathrm{~mm}$. 39 fiber SMA905 connectors are fixed on the fiber bracket. The distance between the neighbour connectors is $15 \mathrm{~mm}$ and all the connectors are in the same height.

The PMTs used in the test platform are manufactured by Beijing Hamamatsu Corp., both of them are wrapped up in the Permalloy film in order to reduce the geomagnetic effect. In the test, the gain of PMTs are set to $3 \times 10^{6}$, and the time transit spread (TTS) is less than $1.0 \mathrm{~ns}$ (FWHM), which is fast enough for the measurement. One PMT (called the test PMT) is installed in the middle of the slide and the height of photocathode center is adjusted as same as the fiber connectors, the distance between the fiber connector and the photocathode is fixed as $4 \mathrm{~mm}$, which could make sure that the outgoing light from the fiber could be entirely detected by the PMT. The other PMT (called the trigger PMT) is installed at the end of the slide, a selected reference optical fiber is fixed just in front of its photocathode as reference fiber.

After the system is adjusted, each fiber takes about 30 seconds for one single test, about 10,000 
triggers are generated and recorded. After one fiber test done, the stepper motor is moved to the position of the next fiber. When the whole 39 fibers tests are done (called a loop), the stepper motor will move reversely for another loop. A whole loop last about 20 minutes, in this mode, the test can be done automatically and continuously. Fig. 3 shows one single measurement of the time offset between one test fiber and reference fiber (the time offset of the channel is not considered in the Fig. 3). The Gaussian function is used to obtain the mean value of a single measurement result.

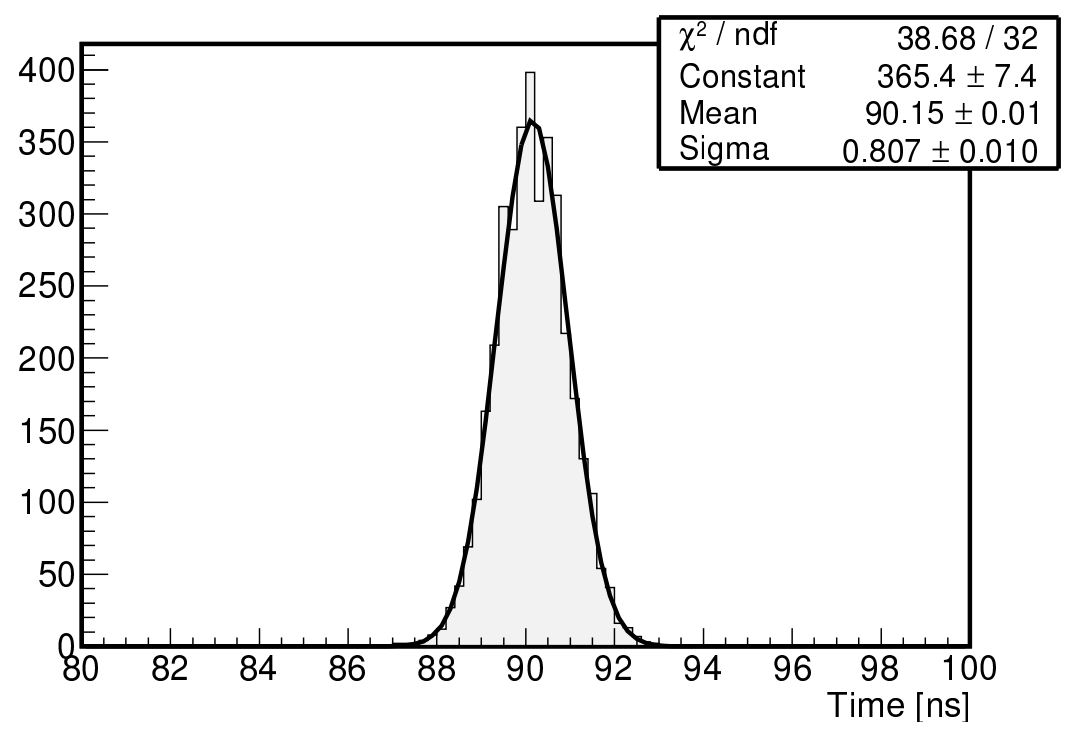

Figure 3: Time offset distribution of a single measurement between the test fiber and reference fiber.

\subsection{Data acquisition}

During the data acquisition, the time offsets between test fibers and the reference fiber, and the intensity of fibers are recorded. After the fiber bundle is deployed and the test platform is initialized, the LED are pulsed by the generator. The trigger PMT and the test PMT signals are firstly sent into FAN-in/FAN-out module split into 2 channels separately, for each PMT, one split signal is sent to FADC for charge measurement, the other firstly sent to ten times amplifier, then goes into the leading edge discriminator, the trigger channel then sent to TDC module as the gate, the test channel then goes to an NIM-ECL level converter, then the ECL signal is sent into TDC test channel. The test hardware, data flow and scheme are shown in Fig. 4. In the test, the LSB of the TDC module (CAEN v775) is set to $35 \mathrm{ps}$, corresponding to $140 \mathrm{~ns}$ time range. Charge measurement uses a $1 \mathrm{GHz}$ sampling rate FADC and its range is $500 \mathrm{mV}$. The threshold of the discriminator is set to $1 / 3$ of the average amplitude of $1 \mathrm{PE}$.

\subsection{Test results}

To study on the performance of the light source and the test platform, three properties are studied: the output uniformity of fibers, time offsets among the fibers and the long-term stability. 


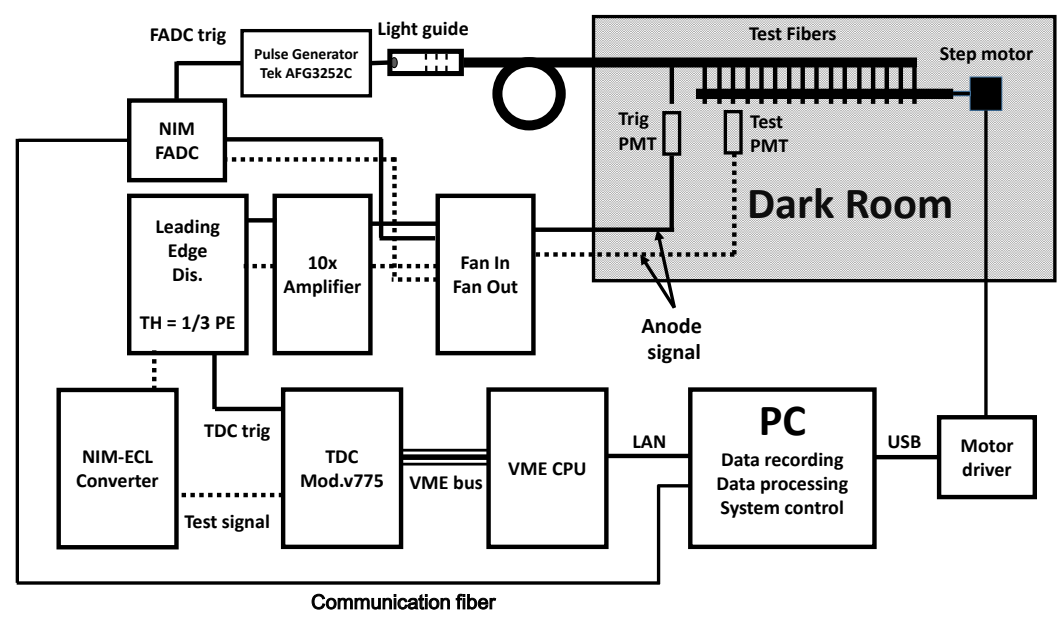

Figure 4: The test flowchart of the relative time offset measurement.

\subsubsection{Light output uniformity}

Different light intensity can lead to difference of arrival time, so called "Q-T" effect, because of the leading edge discriminator used in WCDA. On the contrary, several PEs difference only leads to $10 \mathrm{ps}$ order systematic time offset on the large light intensity. The time calibration should done under large light intensity but also in the linear range of PMT output. In addition, the better consistence of light intensity set in the calibration, the smaller systematic offset due to the "Q-T" effect. In the test, by adjusting the trigger level of LED, the average output of all the 40 optical fibers can be changed from 1 PE to more than 1000 PEs. Fig. 5 shows the relative deviations of output light intensity of 40 optical fibers, when the average value of output intensity is 630.1 PEs. It shows that relative deviations for most of fibers $(\mathrm{N}>36)$ are less than $10 \%$.

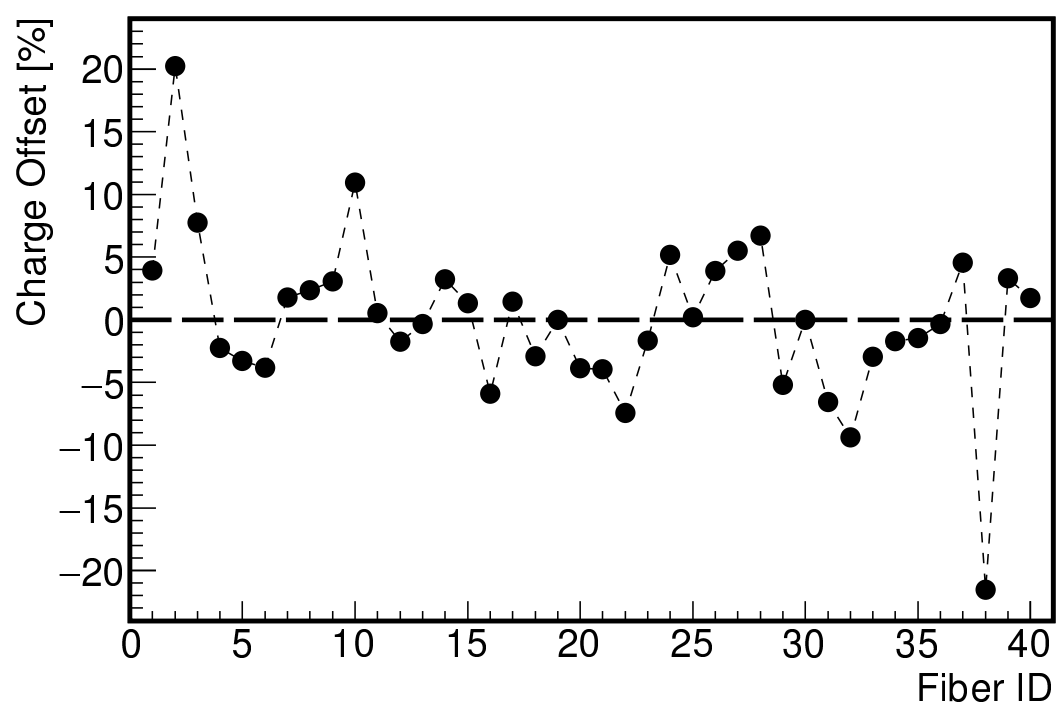

Figure 5: The relative time offsets of the optical fiber bundle. 


\subsubsection{Stability}

During the operation of the detector running, the calibration system will be possibly affected by environment, mainly from the temperature variation and the aging of LEDs. According to the experimental experience of WCDA engineering prototype array [9], the range of the annual temperature variation is about $10^{\circ} \mathrm{C}$. In order to study the robustness and the stability of the calibration light source, most part of fiber $(\geqslant 30 \mathrm{~m})$ and LED light tube are placed outdoors for testing. Besides the environment temperature, the mean light intensity is also set to extreme values. The ambient temperature changes during the test and the average output intensity of the LED is shown in Fig. 6.

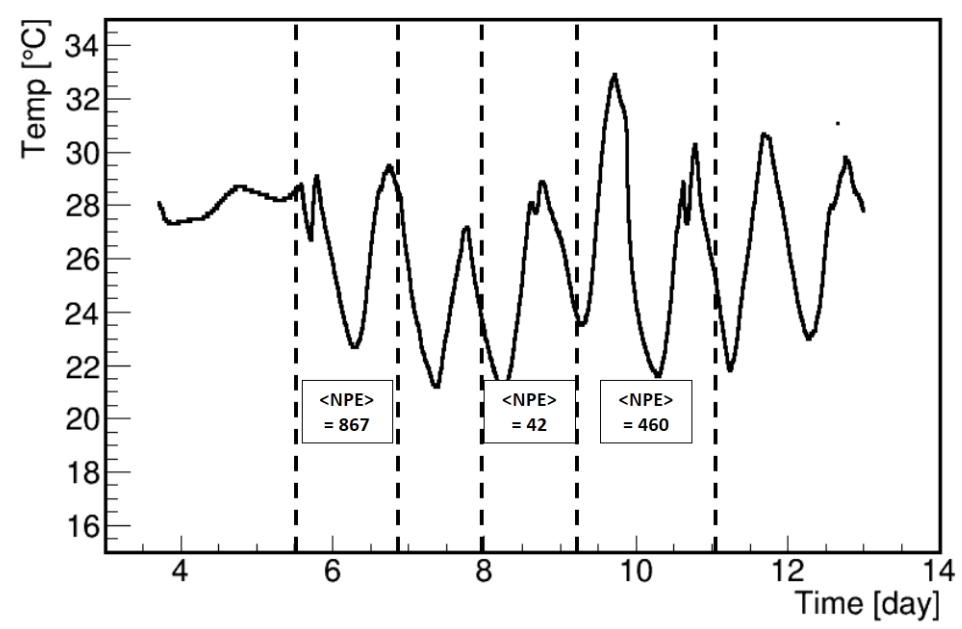

Figure 6: The ambient temperature change along with the average LED output intensity. The average output light intensity of the LED is defined as the average variation value of the output signal of 40 fibers.

For each output intensity condition, the measurement lasts for more than 24 hours to obtain a full temperature cycle. Each cycle contains more than 50 loops of testing, then the mean value of time offsets between the test fibers and reference fiber are obtained, respectively, shown in Fig. 7. The results indicate that the time offsets have very small dependency on the LED light intensity and temperature. By taking $\langle\mathrm{NPE}\rangle=460$ as standard, the maximum deviation of fiber time difference is less than $50 \mathrm{ps}$ when $\langle\mathrm{NPE}\rangle=42$, and the maximum deviation of fiber time difference is $20 \mathrm{ps}$ when $\langle\mathrm{NPE}\rangle=867$. The result shows that the calibration syatem is stable and precision is good enough to do the time calibration. Therefore, it can be applied to the future experiment of WCDA in the long run.

\section{Summary}

According to WCDA detector characteristics, a time calibration light source prototype based on LED and plastic optical fiber bundle is designed, tested by one-dimensional stepping slide and fast PMT fiber time calibration platform. The result shows that the output of the prototype uniformity can be achieved within $\pm 10 \%$ level, and after a long-term test, the system error of the fiber itself is very small, then the optical fiber can be achieved $0.1 \mathrm{~ns}$ time calibration platform below the 


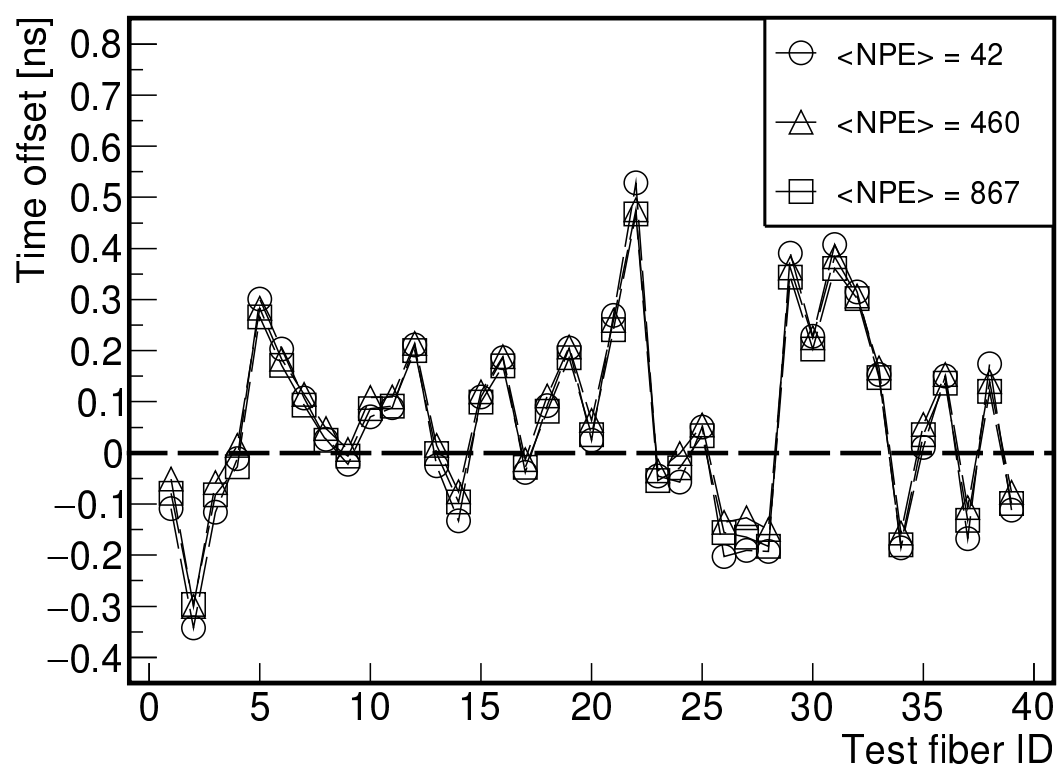

Figure 7: Calibration results for the relative time offsets of the fibers under the three conditions of intensity.

calibration level. The time calibration platform for future optical fiber can be widely used in batch calibration and precise terms of the time calibration.

\section{Acknowledgments}

This work is supported by NSFC No.11375224 and 11675187, Joint Large-Scale Scientific Facility Funds of the NSFC and CAS under contracts No.U1332201 and U1532258, and NSFC of China under contacts No. 11635011.

\section{References}

[1] Z. Cao, Chin. Phys. C (HEP \& NP), 34(2)(2010): 249-252

[2] S. Vernetto, LHAASO collaboration, J. Phys.: Conf. Ser. 718 (2016): 052043

[3] M.J. Chen, et al, R\&D of LHAASO-WCDA. Proceedings of the 32nd ICRC, 2011

[4] Z.G. YAO, et al, LHAASO Collaboration, Design and performance of LHAASO-WCDA experiment, in: Proceedings of 32st ICRC, 2011.

[5] H.A.Ayala Solares, et al, Timing Calibration of the HAWC Observatory, in: Proceedings of the 33rd ICRC, 2013.

[6] H.C. Li, Chin. Phys. C, 37 (1) (2013): 010201

[7] H.H. He, et al, LHAASO collaboration, Design highlights and status of the LHAASO project, in: Proceedings of the 34rd ICRC, 2015.

[8] http://www.bhphoton.com/productinfo.aspx?ProductsId=166\&CateId=212

[9] B. Zhou, et al, LHAASO collaboration , Nucl. Instrum. Methods Phys. Res. A , 724 (2013): 12-19. 